\title{
Clusters of Cognitive and Behavioral Disorders Clearly Distinguish Primary Progressive Aphasia from Frontal Lobe Dementia, and Alzheimer's Disease
}

\author{
C. Marra $^{a} \quad$ D. Quaranta ${ }^{a} \quad$ M. Zinno ${ }^{a} \quad$ S. Misciagna ${ }^{b} \quad$ A. Bizzarro ${ }^{a} \quad$ C. Masullo $^{a}$ \\ A. Daniele ${ }^{a}$ G. Gainotti ${ }^{a}$ \\ ${ }^{a}$ Neuropsychology Service of the Catholic University of Rome, Policlinico Gemelli, and ${ }^{b}$ Fondazione \\ 'Don Carlo Gnocchi', Rome, Italy
}

\section{Key Words}

Frontotemporal dementia $\cdot$ Behavioral psychiatric

symptoms of dementia $\cdot$ Neuropsychological assessment •

Progressive aphasia

\begin{abstract}
Background/Aims: Frontal lobe dementia (FLD) and primary nonfluent progressive aphasia (PnPA) are two forms of frontotemporal lobe degeneration. The relationship between these conditions remains unclear. Our study aimed to better define the behavioral and cognitive clusters characterizing PnPA patients. Methods: We cognitively and behaviorally evaluated three groups of newly diagnosed patients affected by Alzheimer's disease (AD, $n=20), \operatorname{FLD}(n=22)$ and PnPA ( $n=10)$, in order to assess the cognitive-behavioral pattern of PnPA, compared to both FLD and AD. Results: We found, as expected, worse performances in episodic memory in $A D$, of both the verbal fluency and naming tasks in PnPA, while FLD mainly showed behavioral disorders associated with an unremarkable deficit in the executive tasks. PnPA was not characterized by any significant behavioral disorders. Factor analysis-extracted three main factors ('mnesic', 'behavioral' and 'linguistic') clearly correlated to each group. A discriminant analysis based on the extracted factors correctly classified $84.6 \%$ of all patients. Conclusion:
\end{abstract}

The evidence of a characteristics cognitive profile, without any significant behavioral changes, highlights that PnPA is different from other forms of frontotemporal lobe degeneration regarding both the cognitive and behavioral patterns; thus, it should be considered independently in further studies.

Copyright $\odot 2007$ S. Karger AG, Basel

\section{Introduction}

Frontotemporal lobe degeneration (FTLD) is a clinically and neuropathologically heterogeneous condition, which includes different syndromes, sharing a focal neurodegeneration in the anterior brain regions. Several studies have attempted to characterize the various syndromes included in FTLD [1-6]. A distinction between frontal and temporal variants of FTLD according to behavioral and cognitive symptoms has been proposed. Nowadays, the most widely accepted clinical subtypes are a frontal variant (also termed frontal lobe dementia, FLD), a primary nonfluent progressive aphasia (PnPA) and a semantic dementia (SD).

FLD, the most frequent form of FTLD [7], is characterized by a wide range of behavioral disturbances (loss of social cognition, lack of empathy, reduced emotional ex-

Dr. Camillo Marra

Servizio di Neuropsicologia, Università Cattolica/Policlinico Gemelli

Largo A. Gemelli, 8

IT-00168 Roma (Italy)

Tel. +39 063015 4333, Fax +39 063550 1909, E-Mail cmarra@rm.unicatt.it 
pression, disinhibition, apathy, dietary changes, stereotypic and compulsive behaviors $[3,4,6,8]$, which are related to a dysfunction of the anterior, medial and frontoorbital portions of the frontal lobes. In late stages, some patients show the same behavioral alterations as those observed in the Klüver-Bucy syndrome [9]. These marked behavioral abnormalities are frequently associated with cognitive deficits involving 'executive functions' such as planning ability, 'abstraction', set-shifting ability, resistance to interference. Reduction of speech rate and selfinitiated behavior are also frequent [2]. Memory disturbances, when occurring, are usually interpreted as a 'frontal amnesia'. A possible relationship between the site of prevalent atrophy and the behavioral subtypes of FLD (inert/apathetic, disinhibited, and stereotypic) has already been reported $[6,10]$.

Clinically, PnPA is characterized by a nonfluent progressive breakdown of language production [11, 12], with word-finding difficulties and phonological paraphasias, and relatively keeping the other cognitive and behavioral domains for at least another 2 years $[2,11]$. Mutism and agrammatism may occur in later stages $[11,13]$. Recently, PnPA has been reported to have a prognosis worse than Alzheimer's disease (AD) [14]. Although PnPA patients usually do not display severe behavioral abnormalities, depression and frustration are often present. Furthermore, 'frontal' behavior, especially disinhibition [12], has been described in later stages. In PnPA, focal atrophy affects left perisylvian regions, where a metabolic dysfunction may be detected by single-photon emission tomography (SPET) or positron emission tomography [15].

What's more, SD (which corresponds to a fluent form of PPA) is characterized by clear-cut word-finding difficulties and by semantic defects with a progressive disruption of word comprehension, but relative preservation of phonological and syntactic aspects of language, day-today memory and visuospatial functions. Prosopagnosia and semantic difficulties regarding persons and face recognition characterize an SD with a selective right temporal involvement [8].

When all the typical clinical manifestations are present, patients with FLD, SD and PnPA may be quite easily differentiated from patients with $\mathrm{AD}$, especially in the intermediate phases of the illness. However, it is often difficult to distinguish between these clinical entities in other stages of the illness, especially if the diagnosis relies only upon clinical examination and information is gathered through a standard interview. Thus, cognitive and behavioral standardized assessments have proved to be useful in clinical practice.
Several studies have investigated the neuropsychological and behavioral features of AD and FLD. The neuropsychological profile of FLD is usually characterized by an impairment of executive functions [16], at variance with the early deficits of episodic memory and the subsequent impairment of visuospatial abilities which are the neuropsychological hallmarks of AD. However, the results of previous studies were ambiguous, since some investigations reported a substantial overlap between cognitive performance in FLD and AD patients when the overall scores were considered [17-19], while other studies showed contrasting patterns of cognitive impairment such as amnesia/disorientation in AD and executive dysfunction in FLD [20, 21]. Furthermore, test batteries devised to magnify the differences between the neuropsychological profiles of AD and FLD (such as the Frontal Assessment Battery, FAB) failed to provide clear-cut results $[19,22]$. Accordingly, qualitative profiles of neuropsychological and behavioral disturbances might be more helpful in differential diagnosis when compared with raw quantitative neuropsychological measures [23].

Although the patterns of behavioral abnormalities in $\mathrm{AD}$ and FLD have been largely investigated, different assessment methods were used. Some authors used structured or semistructured scales specifically developed to detect behavioral disorders or symptoms which are considered characteristic of FTLD [4, 5, 24, 25]. Alternatively, neuropsychiatric questionnaires such as the Neuropsychiatric Inventory (NPI) [26] have also been utilized $[21,27]$. By means of NPI [27], disregard, apathy, euphoria and aberrant motor activities were mainly manifested by FLD patients, whereas delusions and hallucinations were more common in AD patients, although a significant difference between the two groups was not found regarding this latter behavioral domain. Another study recently reached similar results [21].

All studies aiming at investigating behavioral features by using specific scales or inventories led to similar conclusions. Some authors outlined the importance of social awareness and stereotypic and eating behavior in discriminating frontal and temporal variants of FTLD versus $\mathrm{AD}$ [5]. Interestingly enough, there was no difference in executive dysfunction and self-care, which appeared to be more dependent on the severity of the disease [5]. These findings were substantially replicated by another study, which also included patients affected by vascular dementia [4]. Ritualistic and stereotypic behaviors and eating habits attracted remarkable attention, given their effectiveness in differentiating FTLD from $\mathrm{AD}[24,25]$. 
The difficulties in characterizing the behavioral changes associated with various subtypes of FTLD could derive from differences in the patient samples and in the diagnostic methods used in previous works. Moreover, behavioral abnormalities are common in early stages of FTLD [28], whereas they become more frequent in later stages of $\mathrm{AD}$.

Another source of uncertainties is the collocation of PnPA that represents a peculiar clinical form of FTLD with minor behavioral involvement. Neuropathologically, PnPA could not be early associated with either the frontal or temporal variant of FTLD, while its clinical manifestations have been occasionally linked to SD or to corticobasal degeneration.

The present study was aimed at investigating the cognitive and behavioral abnormalities in patients who show characteristic clinical manifestations of PnPA and frontal lobe dysfunction (FLD) comparing these groups with a group of $\mathrm{AD}$ patients. In particular, we attempted to check the hypothesis that PnPA could be characterized by specific patterns of cognitive performance and by behavioral features which differ from those observed in FLD and AD.

\section{Material and Methods}

\section{Subjects}

A continuous series of 32 patients with clinical diagnosis of FTLD according to the Lund and Manchester criteria [2], at their first evaluation for cognitive impairment, were recruited from among outpatients referring to the Neuropsychology Unit of Policlinico Gemelli in Rome. These patients were compared with a selected group of newly diagnosed patients with clinical diagnosis of probable AD according to the NINDS-ADRDA criteria [29].

Diagnoses were assigned by two independent raters (C.M. and G.G.) on the grounds of the above-cited general criteria, the clinical onset and the neuroradiological findings. Raters were blind to the neuropsychological and behavioral investigations. Three subgroups of frontal variant of FTLD $(n=22)$, PnPA $(n=10)$ and $\mathrm{AD}(\mathrm{n}=20)$ were established.

General raters' criteria for the group assignment were (a) main clinical disorders at the onset: (1) episodic memory difficulties, impairment in learning new information, inability to recall recent events (AD); (2) linguistic disorders characterized by speech slowness, articulatory and word-finding difficulties (PnPA); (3) lack of concern or personality change (FLD). (b) Magnetic resonance imaging (MRI) pattern of atrophy: (1) temporal (mesial) and hippocampal or diffuse cortical atrophy (AD); (2) left perisylvian atrophy (PnPA); (3) prefrontal or frontotemporal atrophy or no cortical atrophy (FLD). (c) SPET hypoperfusion: (1) temporoparietal or parietal and/or posterior cingulated hypoperfusion (AD); (2) left rolandic hypoperfusion (PnPA); (3) frontal or frontotemporal (polar or lateral) hypoperfusion (FLD).
Each patient underwent neurological, neuropsychological and behavioral assessments, cranial MRI and 99Tc-hexamethylpropylene-amine oxime SPET. Due to the several clinical and laboratory investigations, each patient was examined in 2-3 weeks. In all patients, SPET and MRI were carried out closed to the neuropsychological and behavioral assessments.

Patients were enrolled into the study only if the patient had been assigned to the same group by two out of the three criteria; the two judges agreed to such diagnosis; the same judgment was confirmed at a 6 -month follow-up by the same raters through a clinical examination.

General exclusion criteria were: (1) unavailability of neuroradiological or SPECT examinations; (2) unavailability of a sufficiently informed caregiver; (3) a score $<15$ on Mini-Mental State Examination (MMSE).

Eleven patients failed to meet the above-mentioned criteria, so they were excluded from the study. Six patients were excluded because the raters disagreed about their clinical diagnoses, 4 patients met the general criteria for SD but were not considered in the following analyses because they were too few to make comparisons and finally one patient (affected by PnPA) who scored below 15 at MMSE was excluded.

All subjects gave informed consent to participate in the study according the Declaration of Helsinki; the study was approved by the Ethical Committee of the Catholic University of Rome.

Neuropsychological Assessment

Each patient underwent the MMSE and Clinical Dementia Rating (CDR). Furthermore, patients were administered an extensive neuropsychological examination, including tasks of visual and verbal memory (Rey's Auditory Verbal Learning Test, RAVLT, including subtests of immediate and delayed recall and forced-choice recognition [30], Rey's complex figure recall [31], digit span forward and backward [32], Immediate Visual Memory [33]); phonological and semantic verbal fluency (confrontation naming of pictures of objects and actions, copy of Rey's complex figure [31]); executive functions (Temporal Rule Induction task, TRI [34], Stroop test [35], FAB [36], a demanding visual attention task, Multiple Features Targets Cancellation test, MFTC [37]), and finally abstract reasoning (Raven's Colored Progressive Matrices) [38]. As praxis abilities, constructional praxis was evaluated copying pictures with and without landmarks [39]; limb and oral-facial praxis were evaluated by asking the patients to mimic significant and symbolic (e.g. 'send a kiss', 'do the sign of the cross') gestures; two points were assigned when the gesture was performed on oral command, one point if the gesture was performed only on imitation (maximum score: 20).

\section{Neurobehavioral Assessment}

In each patient, behavioral features were assessed by means of NPI, an informant-based 12-item questionnaire [26], by interviewing the patient's primary carer (usually the spouse). The interview assessed the presence, frequency and severity of twelve behavioral symptoms commonly observed in demented patients. For each domain, the interview started with a screening question aimed at assessing the presence of abnormality in a specific behavior. If the caregiver reported an abnormal behavior, this was further explored with more specific questions. Frequency and severity were assessed separately. The total score ranged from 0 (no abnormalities) to 12 (severe abnormalities). 
Table 1. Clinical and demographic characteristics of the patient groups

\begin{tabular}{lcccc}
\hline & $\begin{array}{l}\text { AD } \\
(\mathrm{n}=20)\end{array}$ & $\begin{array}{l}\text { FLD } \\
(\mathrm{n}=22)\end{array}$ & $\begin{array}{l}\text { PnPA } \\
(\mathrm{n}=10)\end{array}$ & $\mathrm{p}$ \\
\hline Age, years & $68.5(8.5)$ & $66.6(7.9)$ & $66.9(8.5)$ & 0.77 \\
Educational level & $8.5(4.59)$ & $9.72(5.57)$ & $10.5(5.21)$ & 0.41 \\
Disease duration, months & $38.5(19.1)$ & $52.7(33.7)$ & $50(33.9)$ & 0.12 \\
MMSE & $18.4(5.8)$ & $19.3(9.1)$ & $21.4(6.8)$ & 0.5 \\
CDR & $3.1(1.2)$ & $2.8(1.3)$ & $2.5(1.0)$ & 0.21 \\
\hline
\end{tabular}

\section{Statistics}

Data were treated with SPSS software for statistics. Demographic and neuropsychological variables were normally distributed. One-way ANOVAs were carried out to analyze demographic variables. The neuropsychological variables were analyzed by means of a one-way MANOVA considering groups as independent variables and the various neuropsychological scores as dependent variables. Within the different multivariate test criteria (Wilks' $\lambda$, Rao's R, Pillai Bartelett Trace), we chose to use in our analysis the Rao's R [40], thus determining the presence of significant effects between the main factor and the interaction among the dependent variables. Post-hoc comparisons between groups were executed by means of Tukey's test for unequal sample size.

Since each of the twelve NPI items was separately analyzed by means of a Kruskal-Wallis ANOVA median test, Bonferroni's correction for multiple comparisons was applied. Since twelve comparisons were made, a p level $<0.004$ after Bonferroni's correction was considered to be statistically significant. A MannWhitney $\mathrm{U}$ test was used to conduct the postcomparisons of the three groups.

The neuropsychological and neurobehavioral variables potentially able to distinguish between the three groups of patients (FLD, PnPA, AD) were treated by means of a principal component analysis (PCA). PCA is a factorial method that allows reduction in the number of variables under study by creating few factors which are linear recombination of the original variables. In this way, it is possible to extract a restricted set of factors accounting for the maximum of the total variance. Three factors were extracted according to the scree plot inspection.

Due to the different pool of behavioral and neuropsychological variables used and to the different distribution of their variance, an Equamax normalized rotation was computed on the first three factors extracted from the PCA. A discriminant analysis with forward stepwise method was also performed in order to identify the variables accounting for the maximum of the total variance (included in the three factors extracted by the PCA) which better allowed us to discriminate the different groups. By means of the same analysis we also checked the number of patients that were correctly classified.

\section{Results}

\section{General Analyses}

Patient groups were comparable for age and educational level, while duration of illness was longer in our FLD group compared with the other groups. The single one-way ANOVA showed no differences on the demographic variables among the three groups as to the overall level of cognitive impairment measured by CDR and MMSE (table 1).

All statistical analyses were carried out on the measures obtained on the first neuropsychological and behavioral examination.

Comparisons between neuropsychological performances in the three groups of patients were made by means of a one-way MANOVA (table 2).

The general multivariate analysis showed a significant global difference between groups (Rao's $\mathrm{R}=2.14$, d.f. $=$ $52,48 ; \mathrm{p}<0.005)$. The specific effect analysis showed that $\mathrm{AD}$ patients scored significantly worse than the other groups on the delayed recall subtest of RAVLT and the recall of Rey's complex figure. Post-hoc comparisons (Tukey's test for unequal sample size) showed that AD and FLD patients were less accurate than PnPA on two measures of attention (accuracy and false alarms on the MFTC test). Moreover, AD patients performed significantly worse than PnPA patients (but not FLD patients) even on tasks of constructional praxis and recognition memory (accuracy and false alarms on the recognition subtest of RAVLT).

The PnPA group performed significantly worse than the other groups on the Phonological Fluency task. Posthoc comparisons showed that PnPA patients performed significantly worse than AD patients on tasks of Semantic Fluency and oral-facial praxis. On these latter tasks, no differences were found between PnPA and FLD groups. 
Table 2. Neuropsychological test comparisons among the three groups of patients

\begin{tabular}{|c|c|c|c|c|c|c|c|}
\hline & \multicolumn{2}{|l|}{$\mathrm{AD}$} & \multicolumn{2}{|l|}{ FLD } & \multicolumn{2}{|l|}{ PnPA } & \multirow[t]{2}{*}{$\mathrm{p}$} \\
\hline & mean & SD & mean & SD & mean & SD & \\
\hline Immediate visual memory & 14.8 & 4.16 & 16.4 & 5.5 & 14.15 & 2.02 & 0.315 \\
\hline \multicolumn{8}{|l|}{ Digit span } \\
\hline Forward & 4.3 & 1.24 & 4.4 & 1.7 & 3.50 & 1.08 & 0.221 \\
\hline Backward & 2.53 & 1.17 & 2.47 & 1.5 & 2.1 & 0.87 & 0.668 \\
\hline \multicolumn{8}{|l|}{ RAVLT } \\
\hline Immediate recall & 17.21 & 8.3 & 21.4 & 14.23 & 23.3 & 9.44 & 0.311 \\
\hline Delayed recall & 0.94 & 1.43 & 3.5 & 3.5 & 5.2 & 3.22 & 0.0006 \\
\hline Accuracy in recognition & 71.4 & 15.87 & 74.5 & 24.56 & 89.4 & 8.92 & 0.055 \\
\hline False alarms in recognition & 11.1 & 7.65 & 9.91 & 8.9 & 4 & 3.16 & 0.055 \\
\hline \multicolumn{8}{|l|}{ Rey's complex figure } \\
\hline Copy & 14.66 & 11.84 & 22.58 & 10.51 & 20.85 & 8.96 & 0.057 \\
\hline Recall & 2.5 & 4.15 & 7.4 & 6.17 & 8.58 & 4.53 & 0.003 \\
\hline Famous face recognition & 21.8 & 6.43 & 21.13 & 7.45 & 26.5 & 4.25 & 0.094 \\
\hline Nouns naming & 21.73 & 6.19 & 18.90 & 9.11 & 20.5 & 8.14 & 0.511 \\
\hline Verbs naming & 16.66 & 6.6 & 13.9 & 6.88 & 17.2 & 8.5 & 0.331 \\
\hline Phonological fluency task & 13.31 & 10.05 & 16.59 & 17.2 & 3.8 & 2.44 & 0.040 \\
\hline Semantic fluency task & 9.21 & 5.01 & 7.57 & 6.9 & 5.2 & 4.16 & 0.20 \\
\hline Raven's progressive matrices ‘ 47 & 15.42 & 8.24 & 18.04 & 7.67 & 20.3 & 4.29 & 0.21 \\
\hline Constructional praxis & 1.84 & 1.24 & 2.4 & 1.11 & 2.85 & 0.75 & 0.052 \\
\hline Constructional praxis with landmarks & 15.31 & 7.57 & 18.63 & 2.46 & 19.7 & 1.88 & 0.037 \\
\hline LIMB praxis test & 17.9 & 1.5 & 16.1 & 4.86 & 17.45 & 2.41 & 0.231 \\
\hline Oral-facial praxis test & 18.56 & 1.76 & 16.2 & 5.61 & 14.1 & 6.47 & 0.050 \\
\hline \multicolumn{8}{|l|}{ MFTC } \\
\hline Accuracy & 72.94 & 19.99 & 78.13 & 18.67 & 91.7 & 4.85 & 0.026 \\
\hline Time & 162.6 & 52.1 & 133.8 & 71.7 & 172.5 & 75.43 & 0.209 \\
\hline False alarms & 12.89 & 16.54 & 12.63 & 15.69 & 2.7 & 2.91 & 0.150 \\
\hline FAB & 9.29 & 3.97 & 8.045 & 6.07 & 9.5 & 3.66 & 0.633 \\
\hline \multicolumn{8}{|l|}{ Stroop test } \\
\hline Interference time & 133.9 & 53.5 & 93.9 & 54.16 & 154.8 & 63.9 & 0.010 \\
\hline Interference errors & 19.32 & 12.31 & 12.6 & 12.4 & 15.4 & 12.28 & 0.214 \\
\hline TRI & 23.03 & 9.8 & 17.4 & 12.23 & 23.8 & 12.2 & 0.192 \\
\hline
\end{tabular}

Values in bold indicate significant differences among groups.

By contrast, the FLD group did not show a specific pattern of neuropsychological impairment. In particular, FLD patients were not significantly more impaired than the other groups in tasks of executive functions (FAB, Stroop, TRI) and were paradoxically faster when executing the Stroop test. The NPI comparisons proved that FLD patients showed more marked behavioral abnormalities when compared to the other groups, since they obtained the highest scores on nearly all NPI subscales (table 3).

In particular, apathy and agitation/aggression were significantly more severe in FLD patients when compared with the other groups. Other behavioral abnormalities (anxiety, euphoria and eating disorders) were more marked in FLD than in PnPA $(\mathrm{p}<0.05)$ patients, whereas no significant differences were found between FLD and $\mathrm{AD}$ groups. PnPA patients were almost unaffected by behavioral disorders apart from a very mild sleep disorder and mildly apathetic behavior. Furthermore in PnPA, no case of delusions, hallucinations and anomalous motor behavior was recorded.

\section{Principal Component Analyses}

In order to identify the neuropsychological or behavioral clusters which may distinguish between the different groups of patients, a principal component factor analysis was carried out with Equamax normalized rotation, given the different canonical distribution of variance 
Table 3. NPI item comparisons among the three groups of patients

\begin{tabular}{|c|c|c|c|c|c|c|c|}
\hline \multirow[t]{2}{*}{ NPI item } & \multicolumn{2}{|l|}{$\mathrm{AD}$} & \multicolumn{2}{|l|}{ FLD } & \multicolumn{2}{|l|}{ PnPA } & \multirow[t]{2}{*}{$\mathrm{p}$} \\
\hline & mean & SD & mean & SD & mean & SD & \\
\hline Delusions & 1.263 & 2.32 & 1.954 & 3.84 & 0 & 0 & 0.267 \\
\hline Hallucinations & 0.526 & 1.42 & 0.227 & 0.61 & 0 & 0 & 0.695 \\
\hline Aggression/agitation & 1.053 & 2.06 & 4.085 & 4.2 & 0.7 & 1.88 & 0.002 \\
\hline Depression & 1.789 & 1.58 & 2.636 & 2.64 & 1.7 & 2.45 & 0.735 \\
\hline Anxiety & 2.158 & 2.89 & 3.273 & 4.10 & 0.2 & 0.63 & 0.03 \\
\hline Euphoria & 0.631 & 2.14 & 1.773 & 3.2 & 0.3 & 0.94 & 0.04 \\
\hline Apathy & 3.684 & 3.0 & 7.636 & 3.23 & 2.5 & 2.75 & 0.0009 \\
\hline Disinhibition & 0.526 & 1.4 & 2.454 & 3.1 & 0.5 & 1.08 & 0.019 \\
\hline Irritability & 2.210 & 2.44 & 4.500 & 4.34 & 1.1 & 1.1 & 0.047 \\
\hline Anomalous motor behavior & 1.684 & 2.7 & 3.182 & 3.9 & 0 & 0 & 0.055 \\
\hline Sleep disorders & 1.579 & 2.69 & 2.773 & 3.14 & 1.7 & 2.6 & 0.18 \\
\hline Eating disorders & 2.368 & 3.5 & 4.727 & 4.43 & 1.1 & 2.6 & 0.022 \\
\hline
\end{tabular}

Values in bold indicate significant differences among groups.

among neuropsychological and behavioral variables. This technique may explain the relationships between observed variables and reduce the same variables to a number of 'main factors', which explain the majority of the variance of the study sample. The Kaiser-MeyerOlkin Measure of Sampling Adequacy (which tests whether the partial correlation was small) was 0.610 , which could be considered a rather good index of reliability of PCA. Three factors accounting for the $52.53 \%$ of the total variance were extracted on the basis of the sloop observed at the scree plot that better described the factor distribution. For each factor, variables were considered to be the most important if their load was greater than 0.70 . The factor loadings for each variable are reported in table 4 .

The three factors included measures strictly belonging to specific cognitive and behavioral domains. Factor 1 (eigenvalue $=11.7$ ), accounting for $30.85 \%$ of the total variance, was related to verbal short-term memory (Digit Span Forward and Backward), linguistic abilities (objects naming), verbal fluency (Phonological and Semantic Verbal fluency) and oral-facial praxis. A one-way ANOVA for this factor showed a significant effect for the diagnostic group $\left(\mathrm{F}_{2-49}=3.01 ; \mathrm{p}<0.05\right)$, with a significant difference between the PnPA (mean score $=-0.62$ ) and $\mathrm{AD}$ (mean score $=0.27$ ) groups, but not between the PnPA and FLD groups (mean score $=-0.04$ ).

The two remaining factors were much weaker, since factor 2 (eigenvalue $=5.08$ ) accounted for $13.37 \%$ of the total variance. This factor was mainly related to perfor- mance on tasks of episodic memory (subtests of delayed recall and recognition accuracy of RAVLT, recall of Rey Osterrieth Figure) and abstract reasoning (Raven's Colored Matrices). A one-way ANOVA carried out on the scores of factor 2 showed a significant effect for the diagnostic groups $\left(\mathrm{F}_{2-49}=10.86 ; \mathrm{p}<0.001\right)$, with a significant difference between $\mathrm{AD}$ (mean score $=-0.64$ ) and both FLD (mean score $=0.21$ ) and PnPA (mean score $=0.81$ ), without a significant difference between FLD and PnPA.

Factor 3 (eigenvalue $=3.16$ ), accounting for the $8.31 \%$ of the total variance, was related to severity of behavioral disorders. Specifically the items Aggression-agitation, Anxiety, Irritability and Aberrant Motor Behavior were significantly loaded in factor 3. A one-way ANOVA for this factor showed a highly significant effect for the diagnostic group $\left(\mathrm{F}_{2-49}=14.69 ; \mathrm{p}<0.001\right)$, with a significant difference between FLD (mean score $=0.67$ ) and both $\mathrm{AD}$ (mean score $=-0.33$ ) and PnPA (mean score $=-0.82$ ), without a significant difference between AD and PnPA.

To explore how the clinical variables identified by the PCA can detect patients belonging to the three groups, a stepwise discriminant analysis was carried out to discriminate the linear combination of the same variables. The stepwise method selected the delayed recall subtest of RAVLT $(<0.001)$, Rey's figure recall $(<0.0001)$, the Phonological and Semantic verbal fluency task $(<0.001)$, Aggression-agitation and Irritability items of NPI as main variables of the analyses ( $\mathrm{F}$ to enter $<0.001$ ). The final classification based on the variables extracted by the 
Table 4. Factor loading of variables extracted by PCA

\begin{tabular}{|c|c|c|c|}
\hline & Factor 1 & Factor 2 & Factor 3 \\
\hline Immediate visual memory & 0.470 & 0.501 & 0.262 \\
\hline \multicolumn{4}{|l|}{ Digit span } \\
\hline Forward & 0.775 & 0.095 & 0.190 \\
\hline Backward & 0.708 & 0.357 & -0.087 \\
\hline \multicolumn{4}{|l|}{ RAVLT } \\
\hline Immediate recall & 0.514 & 0.590 & -0.084 \\
\hline Delayed recall & 0.165 & 0.709 & -0.084 \\
\hline Accuracy in recognition & 0.239 & 0.705 & -0.326 \\
\hline False alarms in recognition & -0.133 & -0.640 & 0.162 \\
\hline \multicolumn{4}{|l|}{ Rey's complex figure } \\
\hline Copy & 0.264 & 0.601 & 0.086 \\
\hline Recall & 0.084 & 0.792 & 0.102 \\
\hline Famous face recognition & 0.378 & 0.542 & -0.350 \\
\hline Nouns naming & 0.744 & 0.104 & -0.203 \\
\hline Verbs naming & 0.644 & 0.175 & -0.260 \\
\hline Phonological fluency task & 0.707 & 0.254 & 0.068 \\
\hline Semantic fluency task & 0.717 & 0.181 & -0.027 \\
\hline Raven's progressive matrices ‘ 47 & 0.253 & 0.738 & -0.045 \\
\hline Constructional praxis & 0.191 & 0.625 & -0.074 \\
\hline Constructional praxis with landmarks & -0.007 & 0.531 & 0.058 \\
\hline Limb praxis test & 0.612 & 0.105 & -0.461 \\
\hline Oral-facial praxis test & 0.702 & -0.173 & -0.259 \\
\hline \multicolumn{4}{|l|}{ MFTC } \\
\hline Accuracy & 0.302 & 0.637 & -0.252 \\
\hline Time & -0.695 & -0.368 & -0.260 \\
\hline False alarms & -0.296 & -0.526 & 0.258 \\
\hline FAB & 0.729 & 0.387 & -0.237 \\
\hline \multicolumn{4}{|l|}{ Stroop test } \\
\hline Interference time & -0.596 & -0.305 & -0.225 \\
\hline Interference errors & -0.622 & -0.566 & -0.073 \\
\hline TRI & 0.478 & 0.192 & -0.421 \\
\hline \multicolumn{4}{|l|}{ NPI } \\
\hline Delusions & -0.126 & -0.309 & 0.569 \\
\hline Hallucinations & 0.077 & -0.384 & -0.051 \\
\hline Aggression/agitation & -0.306 & 0.018 & 0.806 \\
\hline Depression & 0.258 & 0.026 & 0.370 \\
\hline Anxiety & -0.072 & -0.205 & 0.737 \\
\hline Euphoria & -0.356 & 0.244 & 0.608 \\
\hline Apathy & 0.053 & -0.061 & 0.678 \\
\hline Disinhibition & -0.189 & 0.109 & 0.617 \\
\hline Irritability & -0.055 & -0.004 & 0.754 \\
\hline Anomalous motor behavior & -0.234 & -0.341 & 0.709 \\
\hline Sleep disorders & 0.159 & 0.003 & 0.501 \\
\hline Eating disorders & 0.204 & 0.016 & 0.467 \\
\hline
\end{tabular}

Values in bold indicate significant variables in each factor.

stepwise analyses allowed the correct classification of $87.1 \%$ of all patients, ranging from $100 \%$ of PnPA to $77 \%$ of FLD patients. The distribution of correctly classified versus misclassified patients was highly significant $\left(\chi^{2}=\right.$ $16.12, \mathrm{p}<0.001)$.
Table 5. Distribution of patients according to the original and the predicted group membership on the basis of the discriminant analyses

\begin{tabular}{lrrrr}
\hline & FLD & PnPA & AD & $\begin{array}{r}\text { Percent } \\
\text { correct }\end{array}$ \\
\hline FLD $(\mathrm{n}=22)$ & 17 & 2 & 3 & 77.3 \\
PnPA $(\mathrm{n}=10)$ & 0 & 10 & 0 & 100.0 \\
AD $(\mathrm{n}=20)$ & 2 & 1 & 17 & 85.0 \\
\cline { 2 - 5 } Total & 19 & 13 & 20 & 87.1 \\
\hline
\end{tabular}

\section{Discussion}

In our study, we found that specific clusters of behavioral and cognitive defects clearly distinguish between AD, FLD and PnPA patients.

As extensively reported in previous works [41-44], AD patients are correctly identified by tests exploring episodic memory deficits that represent the most reliable neuropsychological hallmark of this disease. Our results support the evidence of an early involvement of episodic memory in the pathological progression of AD.

Also FLD patients usually complain of memory problems, but the organization of memory tracks seems to be the main defect in these patients $[43,44]$. This cognitive aspect is mainly measured by their difficulty in encoding tracks and their reduced learning ability. Our data confirm this. Thus, in a learning task (RAVLT Immediate recall) that is a direct measure of the ability to encode new information, FLD and PnPA perform in the same way as $\mathrm{AD}$. In contrast, episodic long-term memory impairment is mainly reflected by the performance in recall tasks. This domain is chiefly affected in $\mathrm{AD}$ patients so that it could be considered as the crucial cognitive marker in the diagnosis of $\mathrm{AD}$. Therefore, variables of episodic longterm memory were mainly represented in the second factor of the factor analysis, which was strictly related to the diagnosis of $\mathrm{AD}$.

According to some studies, executive dysfunction does not represent the most specific symptom of FLD. Also our FLD group scored the same as AD and PnPA in executive tasks, confirming that the presence of executive disorders is not a specific marker of FLD [19]. The different tools used to explore cognitive abilities usually supported by frontal regions (such as planning or set-shifting tests) could perhaps account for such discrepancies since most of the previous studies employed tasks which assess cognitive functions subserved by dorsal-lateral more

Dement Geriatr Cogn Disord 2007;24:317-326 
than by orbitofrontal circuits, which are preferentially implicated in FLD neuropathological impairment. These considerations could account for the lack of executive tasks in the factor 3 in order to identify FLD patients. Tests of social cognition based on the 'Theory of mind' construct could perhaps be more effective in exploring the earlier stages of FLD [45].

Our data confirm that FLD patients could be more specifically detected by their behavioral disorders than by their cognitive disturbances. Our FLD group presented the most severe behavioral impairment over all the NPI items. Most studies exploring the pattern of neuropsychiatric disorders have shown that apathy represents the most important disorder in FLD [21, 44]. Our data support these investigations confirming that apathy is common and significantly more severe in FLD patients than in PnPA and AD. Nevertheless, apathy is not a specific hallmark of FLD, occurring to a lesser degree in $\mathrm{AD}$ and PnPA patients as well. Accordingly, apathy has not been significantly loaded in the third factor which was associated with the diagnosis of FLD. In addition, sleep disorders and eating disorders linked to the diagnosis of FLD in other studies [5] were not sufficiently represented to be included in the second factor. Actually, qualitative more than quantitative differences characterize the presence of eating disorders in our groups of patients, since appetite was commonly increased in FLD and reduced in $\mathrm{AD}$ and PnPA. By contrast, our FLD patients are correctly identified by aberrant motor behavior, agitation/ aggression, irritability and anxiety symptoms, which are less frequently observed in our AD and PnPA groups.

PnPA seems to be strictly characterized by a pattern of speech and language production disorders which also extend to the orofacial praxis. This pattern was identified by the factor 1 of the PCA. Moreover, our PnPA patients were quite unaffected, apart from the moderate presence of apathy, by behavioral disorders compared to $\mathrm{AD}$ and FLD, probably because the left perisylvian regions could play a secondary role in emotional and social behavior. The presence of apathetic or disinhibited behaviors previously described [10] was not common in our study sample. Our PnPA represents a distinctive group of FTLD about 3 years after the onset of clinical history, characterized by the lack of motor and behavioral disorders.

Even if it has been proposed that PnPA is part of a continuum with other neuropathological conditions such as motor neuron disease and corticobasal degeneration [46], none of our PnPA patients developed motor abnormalities consistent with motor neuron disease or corticobasal degeneration. As compared with other focal degenerative disorders, the prognosis of PnPA seems to be not so severe.

The rather small number of PnPA does not allow us to formulate general conclusions about this group of patients. Nevertheless, the strict selection of a pure group of PnPA, excluding other progressive aphasia, corroborate the consideration that PnPA [13] is clinically different from other subtypes of FTLD because the pattern of impairment concerns almost only the language production mechanisms with a very mild pattern of behavioral abnormalities. Regarding the biological viewpoint, Knibb et al. [47] have recently underlined that PnPA and SD are characterized by an opposite pattern of neuropathological findings, with tau-positive inclusions prevailing in the former and ubiquitin-positive with tau-negative inclusions in the latter. These authors concluded that these two forms should be considered as two different conditions even from a neuropathological viewpoint. Furthermore, Acciarri et al. [48] showed a different pattern of APOE polymorphism in PnPA compared to other FTLD and $\mathrm{AD}$ patients.

Recently, Rosen et al. [49] outlined that neither PnPA nor a logopenic form of PPA are associated with the presence of FLD-like behavior or behavioral disorders similar to those observed in $\mathrm{AD}$ patients. Our data are in agreement with these findings, confirming that PnPA patients are almost free from behavior disorders.

Taking into account both the above-mentioned biological data and our clinical findings, the nosological status of PnPA remains uncertain and cannot be easily included in the dichotomy between frontal and temporal variants of FTLD.

Our study suggests that neuropsychological and behavioral assessments can be helpful in the differential diagnosis of FLD, PnPA and AD, supporting the notion that FTLD can show remarkable heterogeneity of neuropathological, molecular and clinical features.

\section{Acknowledgment}

We acknowledge Ms. M.C. Cullen for the language revision of the manuscript. 


\section{References}

1 Gustafson L: Frontal lobe degeneration of non-Alzheimer type. II. Clinical picture and differential diagnosis. Arch Gerontol Geriatr 1987;6:209-223.

-2 Neary D, Snowden JS, Gustafson L, Passant U, Stuss D, Black S, Freedman M, Kertesz A, Robert PH, Albert M, Boone K, Miller BL, Cummings J, Benson DF: Frontotemporal lobar degeneration: a consensus on clinical diagnostic criteria. Neurology 1998;51: 1546-1554.

$\checkmark 3$ Hodges JR, Miller B: The classification, genetics and neuropathology of frontotemporal dementia. Introduction to the special topic papers. I. Neurocase 2001;7:31-35.

$\checkmark 4$ Bathgate D, Snowden JS, Varma A, Blackshaw A, Neary D: Behaviour in frontotemporal dementia, Alzheimer's disease and vascular dementia. Acta Neurol Scand 2001;103: 367-378.

5 Bozeat S, Gregory CA, Ralph MA, Hodges JR: Which neuropsychiatric and behavioural features distinguish frontal and temporal variants of frontotemporal dementia from Alzheimer's disease? J Neurol Neurosurg Psychiatry 2000;69:178-186.

6 Snowden JS, Bathgate D, Varma A, Blackshaw A, Gibbons ZC, Neary D: Distinct behavioural profiles in frontotemporal dementia and semantic dementia. J Neurol Neurosurg Psychiatry 2001;70:323-332.

7 Rosso SM, Donker Kaat L, Baks T, Joosse M, de Koning I, Pijnenburg Y, de Jong D, Dooijes D, Kamphorst W, Ravid R, Niermeijer MF, Verheij F, Kremer HP, Scheltens P, van Duijn CM, Heutink P, van Swieten JC: Frontotemporal dementia in The Netherlands: patient characteristics and prevalence estimates from a population-based study. Brain 2003; 126:2016-2022.

$\checkmark 8$ Neary D, Snowden J, Mann D: Frontotemporal dementia. Lancet Neurol 2005;4:771780.

9 Cummings JL, Duchen LW: Klüver-Bucy syndrome in Pick disease: clinical and pathologic correlations. Neurology 1981;31:14151422 .

10 Snowden JS, Neary D, Mann DMA: Frontotemporal Lobar Degeneration: Frontotemporal Dementia, Progressive Aphasia, Semantic Dementia. Churchill Livingstone, New York, 1996.

-11 Mesulam MM: Primary progressive aphasia - differentiation from Alzheimer's disease. Ann Neurol 1987;22:533-534.

12 Mesulam MM: Primary progressive aphasia - a language-based dementia. N Engl J Med 2003;349:1535-1542.

- 13 Mendez MF, Clark DG, Shapira JS, Cummings JL: Speech and language in progressive nonfluent aphasia compared with early Alzheimer's disease. Neurology 2003;61: 1108-1113.
14 Le Rhun E, Richard F, Pasquier F: Natural history of primary progressive aphasia. Neurology 2005;65:887-891.

$>15$ Nestor PJ, Graham NL, Fryer TD, Williams GB, Patterson K, Hodges JR: Progressive non-fluent aphasia is associated with hypometabolism centred on the left anterior insula. Brain 2003;126:2406-2418.

16 Mathuranath PS, Nestor PJ, Berrios GE, Rakowicz W, Hodges JR: A brief cognitive test battery to differentiate Alzheimer's disease and frontotemporal dementia. Neurology 2000;55:1613-1620.

17 Gregory CA, Orrell M, Sahakian B, Hodges JR: Can frontotemporal dementia and Alzheimer's disease be differentiated using a brief battery of tests? Int J Geriatr Psychiatry 1997;12:375-383.

18 Siri S, Benaglio I, Frigerio A, Binetti G, Cappa SF: A brief neuropsychological assessment for the differential diagnosis between frontotemporal dementia and Alzheimer's disease. Eur J Neurol 2001;8:125-132.

19 Lipton AM, Ohman KA, Womack KB, Hynan LS, Ninman ET, Lacritz LH: Subscores of the FAB differentiate frontotemporal lobar degeneration from AD. Neurology 2005;65:726-731.

20 Kramer JH, Jurik J, Sha SJ, Rankin KP, Rosen HJ, Johnson JK, Miller BL: Distinctive neuropsychological patterns in frontotemporal dementia, semantic dementia, and Alzheimer disease. Cogn Behav Neurol 2003;16: 211-218.

21 Perri R, Koch G, Carlesimo GA, Serra L, Fadda L, Pasqualetti P, Pettenati C, Caltagirone C: Alzheimer's disease and frontal variant of frontotemporal dementia - a very brief battery for cognitive and behavioural distinction. J Neurol 2005;252:1238-1244.

22 Grossi D, Fragassi NA, Chiacchio L, Valoroso L, Tuccillo R, Perrotta C, Rapone P, Conchiglia G, Trojano L: Do visuospatial and constructional disturbances differentiate frontal variant of frontotemporal dementia and Alzheimer's disease? an experimental study of a clinical belief. Int J Geriatr Psychiatry 2002;17:641-648.

23 Thompson JC, Stopford CL, Snowden JS, Neary D: Qualitative neuropsychological performance characteristics in frontotemporal dementia and Alzheimer's disease. J Neurol Neurosurg Psychiatry 2005;76:920927.

24 Nyatsanza S, Shetty T, Gregory C, Lough S, Dawson K, Hodges JR: A study of stereotypic behaviours in Alzheimer's disease and frontal and temporal variant frontotemporal dementia. J Neurol Neurosurg Psychiatry 2003;74:1398-1402.
25 Ikeda M, Brown J, Holland AJ, Fukuhara R, Hodges JR: Changes in appetite, food preference, and eating habits in frontotemporal dementia and Alzheimer's disease. J Neurol Neurosurg Psychiatry 2002;73:371-376.

26 Cummings JL, Mega M, Gray K, RosenbergThompson S, Carusi DA, Gornbein J: The Neuropsychiatric Inventory: comprehensive assessment of psychopathology in dementia. Neurology 1994;44:2308-2314.

-27 Levy ML, Miller BL, Cummings JL, Fairbanks LA, Craig A: Alzheimer disease and frontotemporal dementias. Behavioral distinctions. Arch Neurol 1996;53:687-690.

28 Lindau M, Almkvist O, Kushi J, et al: First symptoms: frontotemporal dementia versus Alzheimer's disease. Dement Geriat Cogn Disord 2000;11:286-293.

29 McKhann G, Drachman D, Folstein M, Katzman R, Price D, Stadlan EM: Clinical diagnosis of Alzheimer's disease: report of the NINCDS-ADRDA Work Group under the auspices of Department of Health and $\mathrm{Hu}-$ man Services Task Force on Alzheimer's Disease. Neurology 1984;34:939-944.

30 Rey A: Mémorisation d'une serie de 15 mots en 5 répétitions; in Rey A (ed): L'examen clinique en psychologie. Paris, Presses Universitaires de France, 1958.

31 Lezak MD: Neuropsychological Assessment, ed 3. New York, Oxford University Press, 1995

32 Wechsler D: Wechsler Adult Intelligence Scale: WAIS-R Manual. New York, Psychological Corporation, 1981

33 Caltagirone C, Gainotti G, Masullo C, Miceli G: Validity of some neuropsychological tests in the assessment of mental deterioration. Acta Psychiatr Scand 1979;60:50-56.

>34 Villa G, Gainotti G, De Bonis C, Marra C: Double dissociation between temporal and spatial pattern processing in patients with frontal and parietal damage. Cortex 1990;26: 399-407.

35 Caffarra P, Vezzadini G, Dieci F, Zonato F, Venneri A: Una versione abbreviata del test di Stroop: dati normativi nella popolazione italiana. Nuova riv neurol 2002;12:111-115.

36 Dubois B, Slachevsky A, Litvan I, Pillon B: The FAB: a Frontal Assessment Battery at bedside. Neurology 2000;55:1621-1626.

37 Gainotti G, Marra C, Villa G: A double dissociation between accuracy and time of execution on attentional tasks in Alzheimer's disease and multi-infarct dementia. Brain 2001;124:731-738.

38 Raven JC: Progressive Matrices (1947): Sets A, Ab, B: Board and Book Forms. London, HK Lewis, 1949.

39 Gainotti G, Miceli G, Caltagirone C: Constructional apraxia in left brain-damaged patients: a planning disorder? Cortex 1977; 13:109-118. 
40 Lee KL, Harrell FE Jr, Tolley HD, Rosati RA: A comparison of test statistics for assessing the effects of concomitant variables in survival analysis. Biometrics 1983;39:341-350.

-41 Perry RJ, Hodges JR: Differentiating frontal and temporal variant frontotemporal dementia from Alzheimer's disease. Neurology 2000;54:2277-2284.

-42 Pasquier F, Grymonprez L, Lebert F, Van der Linden M: Memory impairment differs in frontotemporal dementia and Alzheimer's disease. Neurocase 2001;7:161-171.

-43 Glosser G, Gallo JL, Clark CM, Grossman M: Memory encoding and retrieval in frontotemporal dementia and Alzheimer's disease. Neuropsychology 2002;16:190-196.
44 Jenner C, Reali G, Puopolo M, Silveri MC: Can cognitive and behavioural disorders differentiate frontal variant-frontotemporal dementia from Alzheimer's disease at early stages? Behav Neurol 2006;17:89-95.

45 Snowden JS, Gibbons ZC, Blackshaw A Doubleday E, Thompson J, Craufurd D, Foster J, Happe F, Neary D: Social cognition in frontotemporal dementia and Huntington's disease. Neuropsychologia 2003;41:688701.

46 Kertesz A: Pick Complex: an integrative approach to frontotemporal dementia: primary progressive aphasia, corticobasal degeneration, and progressive supranuclear palsy. Neurologist 2003;9:311-317.
47 Knibb JA, Xuereb JH, Patterson K, Hodges JR: Clinical and pathological characterization of progressive aphasia. Ann Neurol 2006;59:156-165.

-48 Acciarri A, Masullo C, Bizzarro A, Valenza A, Quaranta D, Marra C, Tiziano FD, Brahe C, Seripa D, Matera MG, Fazio VM, Gainotti G, Daniele A: Apoe epsilon2-epsilon4 genotype is a possible risk factor for primary progressive aphasia. Ann Neurol 2006;59: 436-437.

49 Rosen HJ, Allison SC, Ogar JM, Amici S, Rose K, Dronkers N, Miller BL, Gorno-Tempini ML: Behavioral features in semantic dementia versus other forms of progressive aphasias. Neurology 2006;67:1752-1756. 